\title{
$10 \mathrm{GHz}$ frequency comb spectral broadening in AIGaAs-on-Insulator nano-waveguide with ultra-low pump power
}

\author{
Hu, Hao; Pu, Minhao; Yvind, Kresten; Oxenløwe, Leif Katsuo
}

Published in:

2017 Conference on Lasers and Electro-Optics (CLEO)

Link to article, DOI:

10.1364/CLEO_SI.2017.SW4N.7

Publication date:

2017

Document Version

Peer reviewed version

Link back to DTU Orbit

Citation (APA):

Hu, H., Pu, M., Yvind, K., \& Oxenløwe, L. K. (2017). $10 \mathrm{GHz}$ frequency comb spectral broadening in AIGaAs-onInsulator nano-waveguide with ultra-low pump power. In 2017 Conference on Lasers and Electro-Optics (CLEO) (pp. 1-2). [SW4N.7] IEEE. https://doi.org/10.1364/CLEO_SI.2017.SW4N.7

\section{General rights}

Copyright and moral rights for the publications made accessible in the public portal are retained by the authors and/or other copyright owners and it is a condition of accessing publications that users recognise and abide by the legal requirements associated with these rights.

- Users may download and print one copy of any publication from the public portal for the purpose of private study or research.

- You may not further distribute the material or use it for any profit-making activity or commercial gain

- You may freely distribute the URL identifying the publication in the public portal 


\title{
$10 \mathrm{GHz}$ Frequency Comb Spectral Broadening in AlGaAs- On-Insulator Nano-Waveguide with Ultra-Low Pump Power
}

\author{
Hao Hu, Minhao Pu, Luisa Ottaviano, Elizaveta Semenova, Kresten Yvind and Leif K. Oxenløwe \\ DTU Fotonik, Department of Photonics Engineering, Technical University of Denmark, Building 343, DK-2800 Lyngby, Denmark \\ huhao@fotonik.dtu.dk
}

\begin{abstract}
We experimentally demonstrated $10 \mathrm{GHz}$ frequency comb spectral broadening with a $30-\mathrm{dB}$ bandwidth of $238 \mathrm{~nm}$ in an 11-mm long AlGaAsOI nano-waveguide. The 10-GHz 230-fs pump pulse has an average power of only $12 \mathrm{~mW}$.

OCIS codes: (320.6629) Supercontinuum generation; (190.4390) Nonlinear optics, integrated optics; (230.7370) Waveguide.
\end{abstract}

\section{Introduction}

Optical frequency comb has many applications, such as spectroscopy, metrology, molecular fingerprinting, astronomy, atomic clocks, microwave photonics, and optical communications. There are mainly two ways to generate a broad-band optical frequency comb. One method is to launch a continuous wave (CW) light into a microresonator and a frequency comb can be generated through parametric oscillation [1-6]. However, it suffers from the thermal resonance shift and the absolute frequencies of the generated frequency comb are compromise between Kerr-nonlinear and thermal resonance shifts, therefore a feedback control and a reference laser are usually needed in order to achieve fully frequency stabilization. In addition, the frequency spacing between the generated comb lines is dependent on the free spectral range of the cavity, which has limited accuracy (at $\mathrm{MHz}$ scale); therefore, it's challenging to achieve a frequency comb with exact frequency spacing.

Another way to generate a broad-band optical frequency comb is to spectrally broaden a narrower frequency comb from a pulse laser in a highly nonlinear medium (such as highly nonlinear fibers or photonic crystal fibers) based on self-phase modulation (SPM) and soliton effects [7-9]. Recently, frequency comb spectral broadening (or supercontinuum generation) has also been demonstrated in planar integrated waveguides using different material platforms including silicon [10], chalcogenide [11], silicon nitride [12], and lithium niobate [13]. However, most of the supercontinuum generation in the integrated waveguides have small frequency spacing (usually less than $1 \mathrm{GHz}$ ), which is difficult to be spectrally resolved in a straight-forward manner. It would be advantageous to achieve frequency comb spectral broadening in an integrated waveguide with the frequency spacing between 10 $\mathrm{GHz}$ and $40 \mathrm{GHz}$, which can be both spectrally resolved by a diffraction grating and within electronic bandwidth. One of the main challenges is that the pulses with high repetition rate have low peak power, which is usually not enough to generate broad-band spectral broadening.

Very recently, we have demonstrated AlGaAs-on-insulator (AlGaAsOI) platform as an ultra-efficient nonlinear platform [6, 14-16]. It combines high intrinsic material nonlinearity (on the order of $10^{-17} \mathrm{~W} / \mathrm{m}^{2}$ ), large index contrast between AlGaAs (3.3) and silica cladding (1.5), and low linear and nonlinear losses Error! Reference source not found.. The bandgap of AlGaAs can also be engineered by changing the Al concentration to avoid TPA in the telecom wavelength. In this paper, we achieved $10 \mathrm{GHz}$ frequency comb spectral broadening with a 30-dB bandwidth of $238 \mathrm{~nm}$ (from $1411 \mathrm{~nm}$ to $1649 \mathrm{~nm}$ ) in an 11-mm long AlGaAsOI nano-waveguide. The pump pulse has a pulsewidth of $230 \mathrm{fs}$ and the launched average power is only $12 \mathrm{~mW}$ (peak power of $5.2 \mathrm{~W}$ ). The covered spectral range is more than $\mathrm{C}+\mathrm{L}$ band, which can be potentially used for optical communication as multi-wavelength light sources.

2. $10 \mathrm{GHz}$ frequency comb spectral broadening characterization for AIGaAsOI nano-waveguides

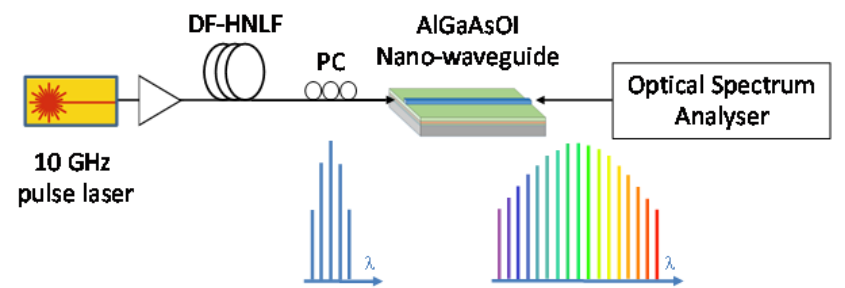

Fig.1 Experimental setup for spectral broadening in an AlGaAsOI nano-waveguide with a cross-section of $290 \times 450$ (or 500 ) $\mathrm{nm}^{2}$. 
(a)

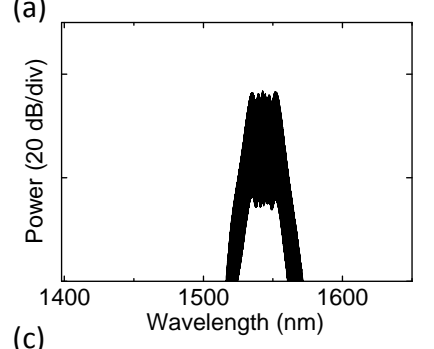

(c)

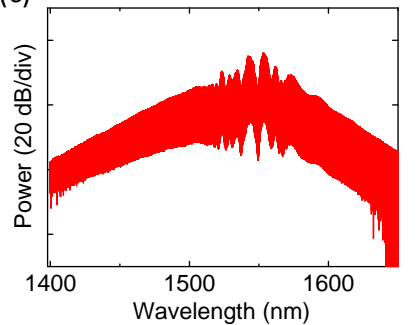

(b)

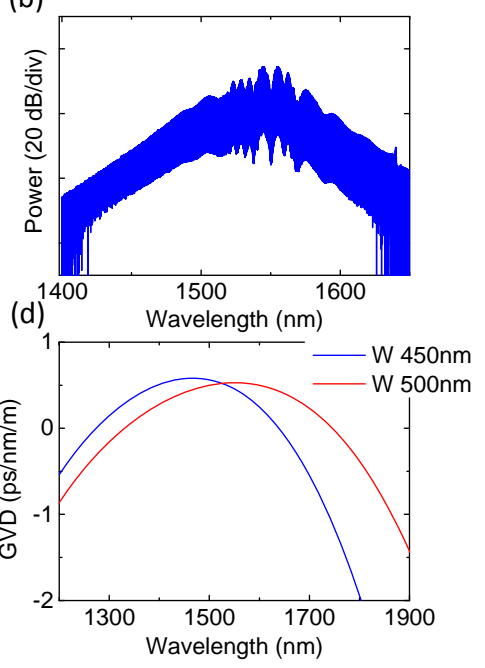

Fig. 2. Measured optical spectra at the input (a) and output of the AlGaAsOI nano-waveguide with the height of $290 \mathrm{~nm}$ and width of $450 \mathrm{~nm}$ (b) and $500 \mathrm{~nm}$ (c); the calculated group velocity dispersion (GVD) for the height of $290 \mathrm{~nm}$ and widths of $450 \mathrm{~nm}$ and $500 \mathrm{~nm}$.

Fig. 1 shows the schematic drawing of the characterization setup for spectral broadening in an AlGaAsOI nanowaveguide. An erbium glass oscillator (ERGO) generates a $10 \mathrm{GHz}$ pulse train at $1550 \mathrm{~nm}$ with picosecond pulse width. The $10 \mathrm{GHz}$ modulation frequency is dependent of a microwave oscillator with an accuracy of $\sim \mathrm{Hz}$ and the absolute frequency of the pulse can be fine-tuned without the need of external reference laser. After amplification in an erbium-doped fiber amplifier (EDFA), the pulses are launched into a dispersion flattened, highly nonlinear fiber (DF-HNLF) to compress the pulses to femto-second pulses (230-fs FWHM). Polarization controller is used to align the pulses to the transverse electric (TE) mode of the waveguide and optical spectrum analyzer (OSA) is used to record the output spectrum. A 290 -nm thick $\mathrm{Al}_{0.17} \mathrm{Ga}_{0.83}$ As film is fabricated on an insulator layer $\left(\mathrm{SiO}_{2}\right)$ through wafer bonding and substrate removal. The width $(450 \mathrm{~nm}$ or $500 \mathrm{~nm}$ ) of waveguide is defined using electron-beam lithography and dry etching. The propagation loss of the waveguide is $\sim 2 \mathrm{~dB} / \mathrm{cm}$. The waveguide under test is 11 $\mathrm{mm}$ long and is tapered to $120 \mathrm{~nm}$ at both sample facets for efficient fiber-to-chip coupling Error! Reference source not found.. Tapered fibers are used at both facets and the coupling loss is $\sim 3 \mathrm{~dB} / \mathrm{facet}$.

Fig.2 (a) shows the spectrum of the 230-fs pump pulse at the input of AlGaAsOI nano-waveguide. Fig.2 (b) and (c) show the spectra at the output of the waveguide with an average pump power of only $12 \mathrm{~mW}$ (peak power of $5.2 \mathrm{~W}$ ), for the waveguide dimension of 290-nm height and 450-nm or 500-nm width, which can be clearly spectral resolved. Fig. 2 (d) shows the calculated group velocity dispersion (GVD) for the 290-nm height and 450-nm or 500-nm width, and both of them exhibits anomalous dispersion at pump wavelength $(1550 \mathrm{~nm})$. For the width of $450 \mathrm{~nm}, 10 \mathrm{GHz}$ frequency comb spectral broadening with a 30-dB bandwidth of $209 \mathrm{~nm}$ (from $1434 \mathrm{~nm}$ to $1643 \mathrm{~nm}$ ) is achieved. For the width of $500 \mathrm{~nm}$, the 30-dB bandwidth of $238 \mathrm{~nm}$ (from $1411 \mathrm{~nm}$ to $1649 \mathrm{~nm}$ ) is achieved. Both dimensions of the waveguide have similar performance, showing a good fabrication tolerance.

\section{Conclusion}

We experimentally demonstrated $10 \mathrm{GHz}$ frequency comb spectral broadening with a 30-dB bandwidth of $238 \mathrm{~nm}$ in an 11-mm long AlGaAsOI nano-waveguide with a cross-section of $290 \times 500 \mathrm{~nm}^{2}$ by launching a 230 -fs pulse train with an average pump power of only $12 \mathrm{~mW}$ (peak power of $5.2 \mathrm{~W}$ ).

\section{Acknowledgement}

The authors acknowledge SPOC research center of excellence (DNRF123) for the support.

\section{Reference}

[1] J. S. Levy et al., Nat. Photonics 4, 37 (2010).

[2] T. Herr et al., Nat. Photonics 8, 145-152 (2014).

[3] L. Razzari et al, Nat. Photonics 4, 41 (2010).

[4] J. Pfeifle et al, Nat. Photonics 8, 375-380 (2014).

[5] V. Brasch et al, Science 351, 357-360 (2016).

[6] M. Pu et al, Optica 3, 823-826 (2016).

[7] P. Russell, Science 299, 358-362 (2003).

[8] H. Hu et al, Opt. Express 19, B343-B349 (2011).

[9] B. P.-P. Kuo et al., J. Lightw. Technol. 31, 3414 (2013).
[10] B. Kuyken et al., Nat. Commun. 6, 6310 (2015).

[11] M. R. Lamont et al., Opt. Express 16, 14938-14944 (2008).

[12] R. Halir et al., Opt. Lett. 37, 1685-1687 (2012).

[13] C. R. Phillips et al., Opt. Lett. 36, 3912-3914 (2011).

[14] M. Pu et al., OFC 2015, postdeadline paper Th5A.3 (2015).

[15] H. Hu et al., CLEO 2016, postdeadline paper JTh4C.1 (2016).

[16] M. Pu et al., CLEO 2016, paper AM3J.3 (2016).

[17] M. Pu et al., Opt. Commun. 283, 3678-3682 (2010). 